\title{
Multinodular adult rhabdomyoma in the base of the tongue excised conservatively using submental midline approach with hyoid bone split: A case report
}

\author{
Hiroyuki Harada ${ }^{1}$, Shogo Shinohara ${ }^{1}$, Masahiro Kikuchi ${ }^{1}$, Keizo Fujiwara ${ }^{1}$, Atsushi Suehiro ${ }^{1}$, I ppei \\ Kishimoto ${ }^{1}$, Fumihiko Kuwata ${ }^{1}$, Ryosuke Yamamoto ${ }^{1}$, Kazuki Hayashi ${ }^{1}$, Yukihiro I mai ${ }^{2}$, Yasushi \\ Naito $^{1}$
}

1. Department of Otolaryngology-Head and Neck Surgery, Kobe City Medical Center General Hospital, 2-1-1 MinatojimaMinamimachi, Chuo-ku, Japan. 2. Department of Clinical Pathology, Kobe City Medical Center General Hospital, 2-1-1 Minatojima-Minamimachi, Chuo-ku, Japan.

Correspondence: Hiroyuki Harada, MD. Address: Department of Otolaryngology-Head and Neck Surgery, Kobe City Medical Center General Hospital Minatojima-Minamimachi 2-1-1, Chuo-ku, Kobe 650-0047, Japan.

E-mail: ntybt959@ybb.ne.jp

Received: August 30, 2015

Accepted: October 7, 2015

Online Published: October 15, 2015

DOI : $10.5430 /$ crcp.v2n4p89

URL: http://dx.doi.org/10.5430/crcp.v2n4p89

\section{Abstract}

Background: Adult rhabdomyoma (ARh) is a rare benign tumor of skeletal muscle origin. ARh occurs mainly in the head and neck region. This report details the surgical excision of a large multinodular ARh in the base of the tongue that was causing severe sleep apnea.

Findings: Magnetic resonance imaging revealed that the base of the tongue was almost completely replaced by a $6 \mathrm{~cm} \times$ $8 \mathrm{~cm}$ tumor.

Methods: Conservative surgical excision was done by submental midline approach with hyoid bone split.

Results: Surgery improved the patient's sleep apnea without damaging the swallowing function. The patient was able to secede from nasal continuous positive airway pressure and has remained symptom-free for 12 months postoperatively.

Conclusions: This newly designed approach is suitable for excision of benign tumors in the base of the tongue to obtain a patent airway without damaging the function of the tongue base.

\section{Key words}

Adult rhabdomyoma, Obstructive sleep apnea, Conservative excision, Submental midline approach with hyoid bone split

\section{I ntroduction}

Rhabdomyomas $(\mathrm{Rh})$ are benign mesenchymal tumors with skeletal muscle differentiation; these are classified into cardiac and extracardiac Rh types based on location. Extracardiac Rh is further classified into adult, genital, or fetal types depending on the degree of differentiation, although some overlap exists ${ }^{[1]}$. Adult rhabdomyomas (ARh) are relatively 
rare; $80 \%$ of them arise in the head and neck region, mainly in the oral cavity, pharynx, and larynx ${ }^{[1]}$. ARh usually present with no symptoms, but some symptoms including upper airway obstruction may appear depending on the tumor site and volume $^{[2,3]}$.

This article presents a case of multinodular ARh originating in and occupying the base of the tongue, causing severe sleep apnea. We developed a submental midline approach with hyoid bone split for conservative surgical excision of ARh in the base of the tongue; this newly designed method has not been previously described in the literature.

\section{Case presentation}

A 73-year-old man presented with several years' history of slowly advancing sleep apnea and muffled voice caused by a mass in the base of the tongue. He had a transoral biopsy of the tumor when he was 51 years old, and received a histopathological diagnosis of multinodular ARh. The patient was treated with a wait-and-see policy over the next 20 years as the tumor was benign and existed deep in the base of the tongue. Eventually, he developed severe obstructive sleep apnea that could not be controlled by nasal continuous positive airway pressure (CPAP), and was referred to our department for tracheostomy.

Physical examination revealed a soft, painless mass in the submental region, pushing up the floor of the mouth. Fiberscopic examination revealed a bulky mass covered with normal mucosa in the base of the tongue, severely overhanging the larynx and narrowing the airway.

Magnetic resonance imaging (MRI) revealed that the base of the tongue was almost completely replaced by a $6 \mathrm{~cm} \times 8 \mathrm{~cm}$ tumor, which had a slightly higher intensity than muscle in both T1 and T2 weighted images and was weakly enhancing (see Figure 1). The extrinsic muscles were completely missing in the tumor, and the suprahyoid muscles were pushed out and appeared thinner, especially in the left side.

Conservative surgical excision was aimed at gaining a patent airway to enable the patient to secede from CPAP without damaging the remaining tongue function. The patient underwent a tracheotomy under local anesthesia prior to surgery to safely secure the airway. A horizontal skin incision was made just above the hyoid bone. After retracting the skin flap to expose the surgical field from the lower edge of mandible to the upper edge of the cricoid, an approach to the base of the tongue was established by dividing the midline of the supra- and infra-hyoid muscles with a hyoid bone split (see Figure 2). By retracting the midline to both sides, the tumor nodules were able to be removed manually piece by piece without damaging the remaining extrinsic muscle fibers. A secondary approach was made by retracting the left mylohyoid muscle medially to remove the tumor protruding under the left submandibular gland. We removed as much of the tumor as possible, taking special care not to perforate the mucosa of the pharynx. After tumor removal, the divided midline was sutured closed with absorbable 3-0 thread. The total tumor weighed 84.25 g. Macroscopically, the specimen consisted of multiple red-brown, soft, lobulated nodules similar to veal liver (see Figure 3). Histological examination of the recurrent tumor revealed compact growth of polygonal multinucleated cells. Their cytoplasm were deeply eosinophilic with irregularly oriented cross striation, or vacuolated. Evenly sized round nuclei with small nucleoli were mostly thrust aside. Mitotic figure was rare (see Figure 4A). Immunohistochemistry showed positivity for desmin (see Figure 4B) and actin. Ki-67 labeling index was $0.1 \%$.

Immunohistochemical staining demonstrated that the tumor cells had strong reactivity to antibodies directed against muscle specific actin and desmin (see Figure 4B).

The patient was able to take oral fluids and solids on the first postoperative day, and was discharged with a tracheostomy tube on the eleventh postoperative day. After closure of the tracheostoma 2 months after surgery, the sleep apnea improved 
enough to let him secede from CPAP. MRI examination 2 months after surgery showed clearance of the airway (see Figure 5). The patient has maintained this condition without symptoms for over 12 months.
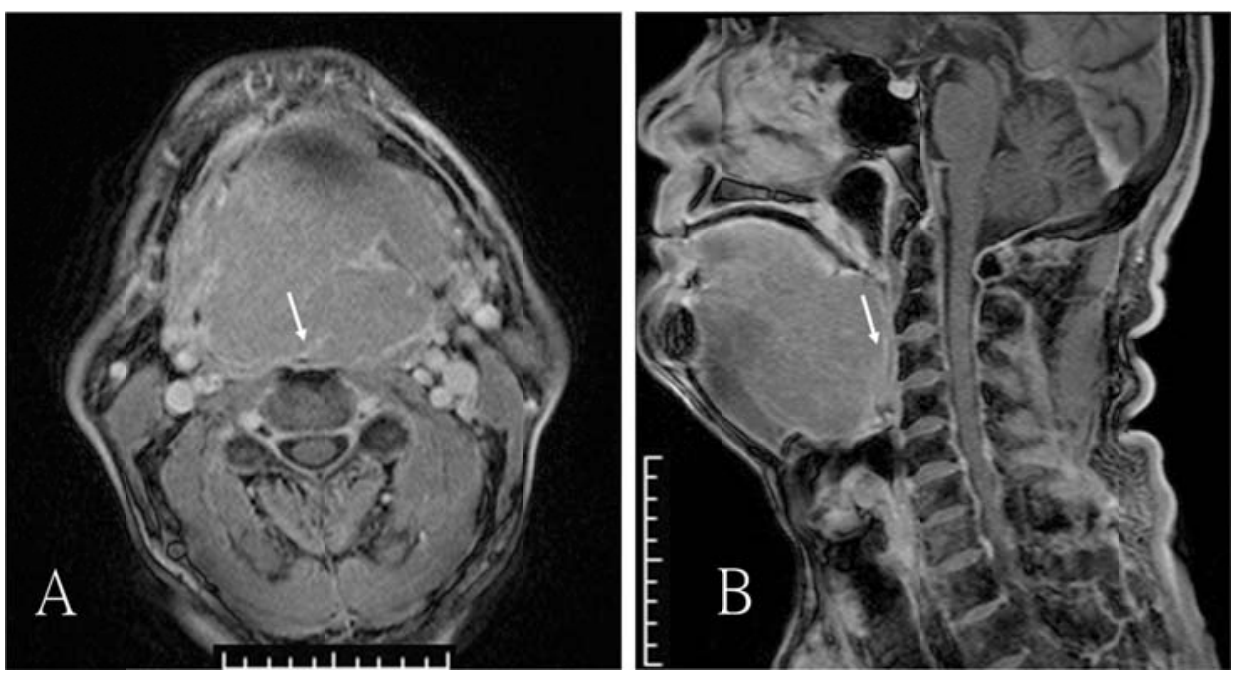

Figure 1. Preoperative enhanced MRI images (A. axial, B. coronal). A weakly enhancing mass occupied the base of the tongue, effacing the upper airway (arrows).

Figure 2. View during the operation. View during the operation demonstrating the space obtained by submental midline approach by hyoid bone split. T: tumor, * : split pieces of the hyoid bone.

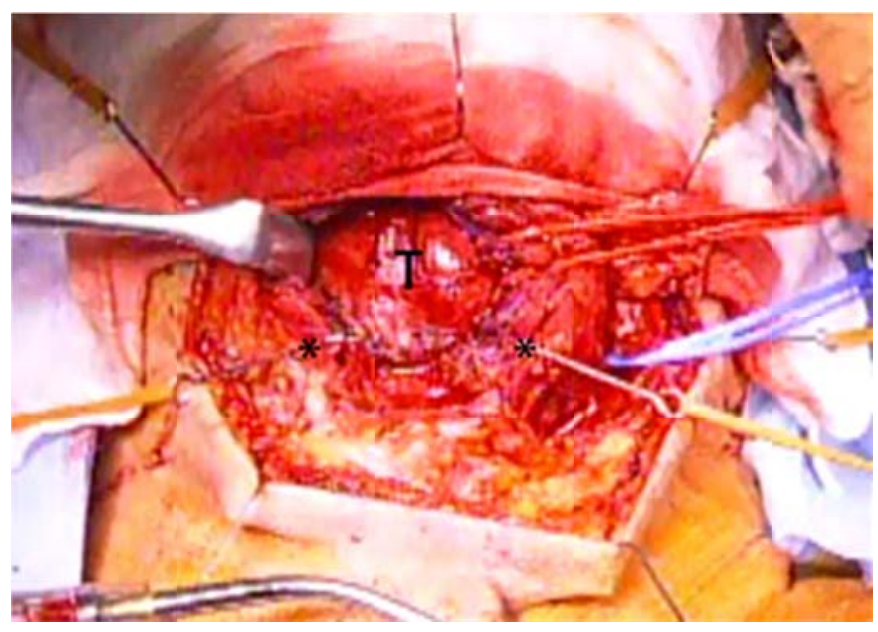

Figure 3. Macroscopic findings of the tumor. Macroscopic findings of the dissected specimen after formalin fixation. The tumor was multinodular and looked similar to veal liver.

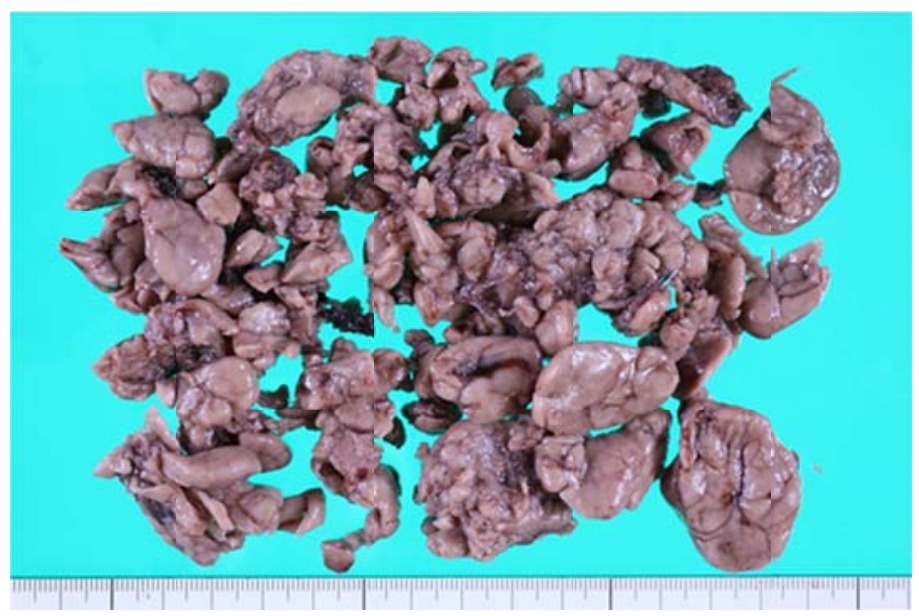



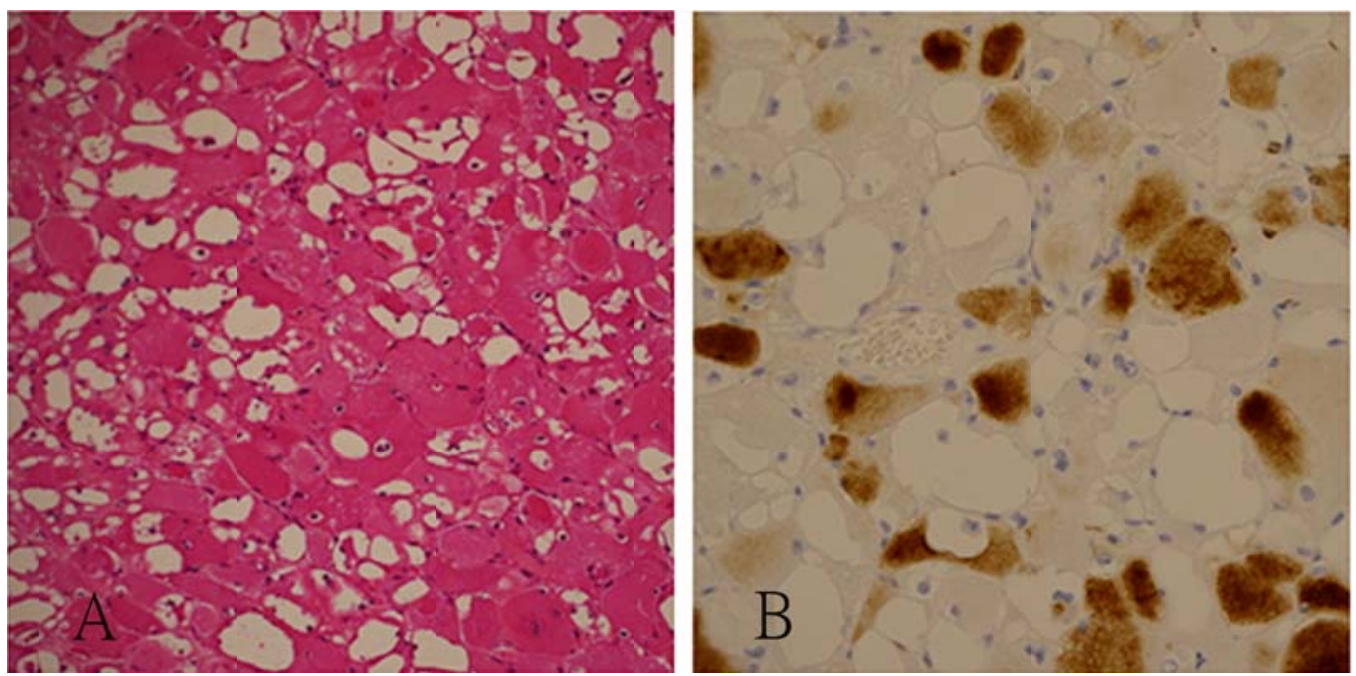

Figure 4. Microscopic findings of the tumor. A: Haematoxylin and Eosin $(H \&$ E) stain $(\times 100)$ of the excised specimens showed large polygonal cells with eosinophilic and vacuolated cytoplasm. B: Brown positive staining of desmin immunohistochemistry highlighted cross striation.
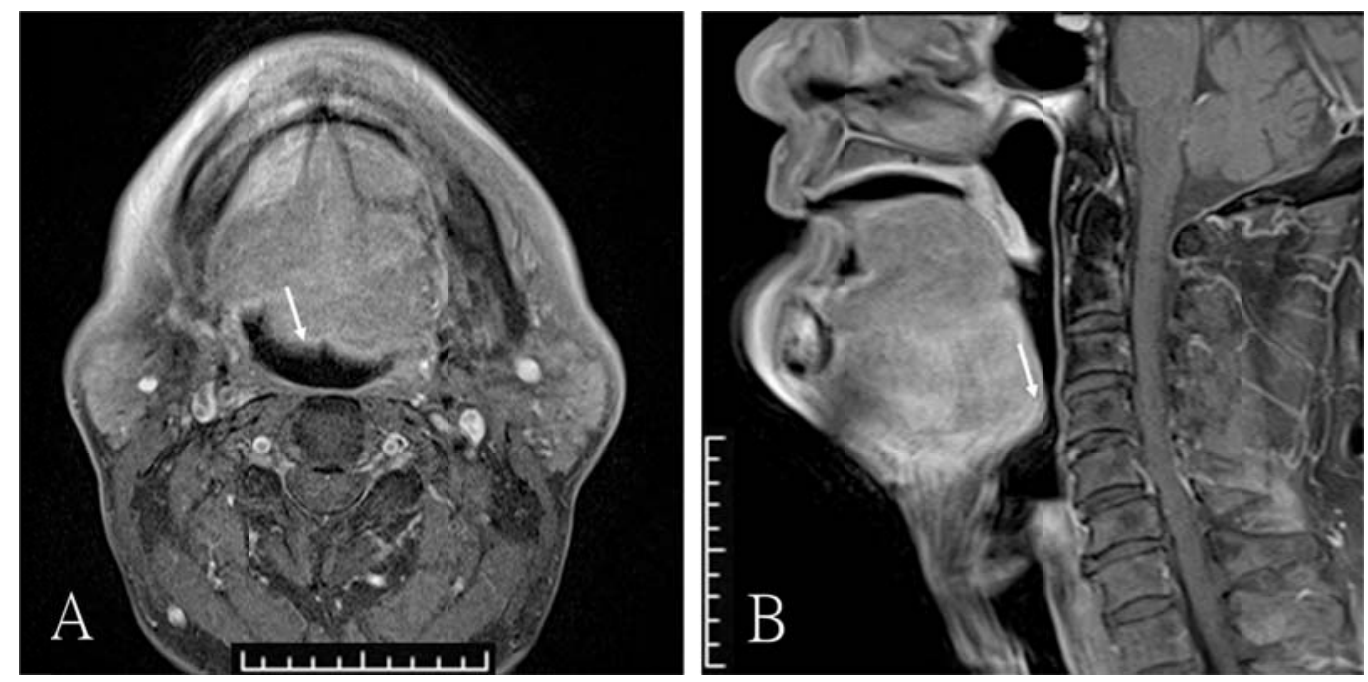

Figure 5. Enhanced MRI images 2 months after conservative excision of the tumor. A is axial and B is coronal. The mass was reduced in size and the airway was clear (arrows).

\section{Discussion}

ARh is more common in the head and neck region than any other anatomic site, but it is still a rare neoplasm of the oral cavity ${ }^{[4,5]}$. Fetal Rh was first reported on the tongue in 1897; however, this is considered to be a development lesion, not a "true neoplasm" ${ }^{33}$. Epidemiologically, most patients with ARh are 40 to 70 years old (mean age 50 years) and predominantly male (ratio 3-5:1), with no predilection for a particular race ${ }^{[1]}$. Surgical excision is the treatment of choice for ARh.

Pathologically, cytoplasmic cross striation and desmin positivity confirmed skeletal muscle differentiation and excluding other eosinophilic soft tissue tumors such as granular cell tumor, hibernoma, oncocytoma and rhabdoid tumor.

Tumor recurrence was reported in $16 \%-42 \%$ of cases in the literature, with most recurrences caused by incomplete removal or the multicentric nature of the tumor ${ }^{[6-8]}$. Recurrence may develop after many years or decades as these tumors 
grow extremely slowly ${ }^{[8]}$. Malignant transformation has basically not occurred, and hence conservative surgical excision is a valid treatment option ${ }^{[1,5,9,10]}$. On the other hand, some literature reported malignant transformation ${ }^{[11]}$. Therefore, analysis of cell proliferation markers such as Ki-67 and the follow-up observation are needed.

In the present case, the tumor grew gradually over 22 years. Previous pathological findings demonstrated that the tumor was multinodular. MRI examination revealed that the extrinsic muscles were completely missing in the tumor, indicating that the tumor nodules were scattered between the muscle fibers.

Conservative surgical excision aimed at improving the severe obstructive sleep apnea without damaging the tongue function was deemed appropriate considering the patient's future quality of life. A newly designed approach to the base of the tongue via submental midline approach with hyoid bone split was used. This approach creates a wider space on the midline between both sides of the supra- and infra-hyoid muscles without damaging the muscles themselves, the motor or sensory neural networks, and/or blood supply to the supra- and infra-hyoid muscles and extrinsic muscles. As a result, the patient took in fluids and solids orally just 1 day after surgery. This is a report of a case and fortunately the patient did well, however our surgical method can be an option because the efficacy of this minimally invasive method was proven.

\section{Conclusion}

ARh in the base of the tongue was conservatively excised by submental midline approach with hyoid bone split to improve sleep apnea. This newly designed approach is suitable for excision of benign tumors in the base of the tongue to obtain a patent airway without damaging the function of the tongue base.

\section{References}

[1] Gnepp AR. Diagnostic surgical pathology of the head and neck. Philadelphia: Saunders. 2009; 251.

[2] Pichi B, Manciocco V, Marchesi P, et al. Rhabdomyoma of the parapharyngeal space presenting with dysphagia. Dysphagia. 2008; 23: 202-4. PMid: 17965913. http://dx.doi.org/10.1007/s00455-007-9125-0

[3] Papaspyrou G, Werner JA, Roessler M, et al. Adult rhabdomyoma in the parapharyngeal space: report of 2 cases and review of the literature. American journal of otolaryngology. 2011; 32: 240-6. PMid: 20392534. http://dx.doi.org/10.1016/j.amjoto.2010.01.007

[4] Neville BW, McConnel FM. Multifocal adult rhabdomyoma. Report of a case and review of the literature. Archives of otolaryngology. 1981; 107: 175-8. http://dx.doi.org/10.1001/archotol.1981.00790390041012

[5] Khan AM, Chor PJ, Eisenbeis JF. Adult extracardiac rhabdomyoma of the parapharyngeal space. Ear, nose, \& throat journal. 2012; 91: 7-10. PMid: 22996720.

[6] Corio RL, Lewis DM. Intraoral rhabdomyomas. Oral surgery, oral medicine, and oral pathology. 1979; 48: 525-31. http://dx.doi.org/10.1016/0030-4220(79)90299-8

[7] Amelia SA, de Araujo VC, Passador SF, et al. Intraoral adult rhabdomyoma: a case report. Case reports in dentistry. 2013; 741548. http://dx.doi.org/10.1155/2013/741548

[8] Kapadia SB, Meis JM, Frisman DM, et al. Adult rhabdomyoma of the head and neck: a clinicopathologic and immunophenotypic study. Human pathology. 1993; 24: 608-17. http://dx.doi.org/10.1016/0046-8177(93)90240-H

[9] Zacharia TT, Som PM. Multiple adult rhabdomyomas of the oropharynx, base of the tongue, and floor of the mouth: magnetic resonance findings. Archives of otolaryngology-head \& neck surgery. 2008; 134: 892-4. PMid: 18711066. http://dx.doi.org/10.1001/archotol.134.8.892

[10] Maglio R, Francesco S, Paolo M, et al. Voluminous extracardiac adult rhabdomyoma of the neck: a case presentation. Case Rep Surg. 2012; 10: 1155. http://dx.doi.org/10.1155/2012/984789

[11] Cuesta G, Luengo MFA, Vila JN. Rhabdomyoma of the base of the tongue. A case report. Int J Oral Maxillofac Surg. 2000; 29: 136-7. http://dx.doi.org/10.1034/j.1399-0020.2000.290212.x 